Research Paper

\title{
Survival and Toxicities of IMRT Based on the RTOG Protocols in Patients with Nasopharyngeal Carcinoma from the Endemic Regions of China
}

Shao-Bo Liang1,2*, Yan Wang ${ }^{3 *}$, Xue-Feng Hu${ }^{1}$, Sha-Sha He ${ }^{2}$, Xing-Li Yang ${ }^{2}$, Li-Zhi Liu ${ }^{2}$, Chun-Yan Cui ${ }^{2}$, Yong $\mathrm{Chen}^{3 凶}$, Li-Wu Fu${ }^{2 \bowtie}$

1. Department of Radiation Oncology, Cancer Center, First People's Hospital of Foshan Affiliated to Sun Yat-sen University, Foshan 528000, China;

2. Sun Yat-sen University Cancer Center; State Key Laboratory of Oncology in South China; Collaborative Innovation Center for Cancer Medicine, Guangzhou 510060, China;

3. Department of Radiation Oncology, The First Affiliated Hospital of Sun Yat-Sen University, Guangzhou, China.

* These authors contributed equally to this work.

$\triangle$ Corresponding authors: Li-Wu Fu, State Key Laboratory of Oncology in South China, Cancer Center, Sun Yat-sen University, Collaborative Innovation Center for Cancer Medicine, Guangzhou 510060, China. Telephone: +86-20-87343163 Fax: +86-20-87343392 E-mail: Fulw@mail.sysu.edu.cn Yong Chen, Department of Radiation Oncology, The First Affiliated Hospital of Sun Yat-Sen University, Guangzhou 510080, China Telephone: +86-20-87755766-8425 Fax: +86-20-87330736 E-mail: chenyong@mail.sysu.edu.cn

(C) Ivyspring International Publisher. This is an open access article distributed under the terms of the Creative Commons Attribution (CC BY-NC) license (https://creativecommons.org/licenses/by-nc/4.0/). See http://ivyspring.com/terms for full terms and conditions.

Received: 2017.03.31; Accepted: 2017.08.04; Published: 2017.10.17

\begin{abstract}
Background: This study evaluated the survival outcomes and toxicities of intensity-modulated radiation therapy (IMRT) based on the RTOG 0225/0615 RT protocols in patients with nasopharyngeal carcinoma (NPC) from a region of China where this tumor type is endemic.

Methods: A total of 455 patients with non-metastatic, histologically-confirmed NPC were retrospectively reviewed. All patients were treated by IMRT using the RTOG 0225/0615 RT protocols; $91.1 \%(288 / 316)$ of patients with stage III-IVb NPC received concurrent chemotherapy +/- induction chemotherapy or adjuvant chemotherapy.

Results: Estimated four-year overall survival (OS), failure free survival (FFS), local relapse free survival (LRFS), regional relapse free survival (RRFS) and distant metastasis free survival (DMFS) were 83.8\%, 80.5\%, 94.3\%, $96.7 \%$ and $85.8 \%$, respectively. T and $\mathrm{N}$ category were significant prognostic factors for OS, FFS, RRFS and DMFS; and T category, for LRFS. In-field failure was the major loco-regional failure pattern. During RT, 206 (45.3\%) patients experienced acute grade 3-4 toxicities. The most common acute toxicity was mucositis; 124 (27.2\%) patients experienced grade 3-4 mucositis; 46 (10.1\%) experienced serious late toxicities. The most common late toxicity was MRI-detected radiation-induced temporal lobe necrosis $(6.8 \%)$.

Conclusions: The RTOG IMRT protocols are feasible for patients with NPC from the endemic regions of China.
\end{abstract}

Key words: Nasopharyngeal carcinoma; intensity-modulated radiotherapy; RTOG 0225; RTOG 0615.

\section{Introduction}

Unlike other carcinomas of the head and neck, nasopharyngeal carcinoma (NPC) has an extremely unbalanced incidence. NPC is rare $\left(0.5 / 10^{5}\right.$ cases among males per year) in the majority of white populations but is more common in China, with an incidence of $20-50 / 10^{5}$ males per year in southern China $^{1}$. In these endemic areas, more than $95 \%$ of cases are non-keratinizing NPC ${ }^{2}, 3$, which is sensitive to radiotherapy (RT) and chemotherapy. Therefore, $\mathrm{RT}$ is the main treatment method for non-metastatic NPC and concurrent chemotherapy is recommended for local-regionally advanced NPC ${ }^{4-7}$.

Intensity-modulated radiation therapy (IMRT) is commonly used worldwide to treat NPC. Compared to two-dimensional RT (2-DRT) and threedimensional conformal RT (3-DCRT), IMRT provides 
a higher and more conformal radiation dose while effectively decreasing the dose to surrounding normal tissues. Therefore, IMRT not only results in excellent local control in NPC, but also significantly reduces RT-related late toxicities ${ }^{8-11}$. However, the design of the RT plan is one of the most important components of treatment for NPC.

Both protocols devised by the Radiation Therapy Oncology Group (RTOG), RTOG 0225 and RTOG 0615, recommend a detailed RT plan for NPC. These trials resulted in excellent loco-regional control and encouragingly low rates of grade 3-4 acute toxicities in patients from the non-endemic regions ${ }^{12,13}$. Therefore, the RTOG RT plan has been widely accepted 14,15 . However, the reduced-target-volume IMRT (RTV-IMRT) RT plan is also widely used in the regions of China where NPC is endemic ${ }^{16}$. The RTV-IMRT and RTOG RT plans have different prescribed doses, single RT doses and clinical target volume (CTV) delineation strategies, as well as other variations. Follow-up studies have confirmed the RTV-IMRT provides favorable treatment outcomes with acceptable toxicities in patients from endemic regions 17,18 .

However, there are few reports on application of the RTOG RT protocols in patients from the regions of China where NPC is endemic. Therefore, we initiated a retrospective study of a large cohort of patients from an endemic region of China to evaluate the survival outcomes and toxicities of the IMRT treatment plan of the RTOG 0225 and RTOG 0615 protocols.

\section{Materials and Methods}

\section{Study population}

The Institutional Review Board of First People's Hospital of Foshan Affiliated to Sun Yat-sen University approved this retrospective analysis of clinical routine data; an exemption from requiring written informed consent was granted. All 455 consecutive patients with newly-diagnosed, histologically-proven, non-metastatic NPC treated by IMRT based on the RTOG 0225/0615 RT protocols at our cancer center between April 2010 and March 2014 were included. All cases had the non-keratinizing pathological type. The cohort included 347 males and 108 females (male:female ratio, 3.2:1), with a median age of 45 years (17-80 years).

Pretreatment examination included medical history, physical examination, hematology and biochemistry profiles, electrocardiogram, chest X-ray, abdominal ultrasound, bone scan, nasal endoscopy and biopsy, and magnetic resonance imaging (MRI) scan of nasopharynx and neck. All patients were restaged according to the 8th edition of the American
Joint Commission on Cancer staging system (AJCC) ${ }^{19}$. The stage distribution for the entire cohort was: $124 / 455$ (27.3\%) in T1, 63 (13.8\%) in T2, 155 (34.1\%) in T3, and $113(24.8 \%)$ in T4; $58(12.7 \%)$ in N0, 258 $(56.7 \%)$ in N1, 110 (22\%) in N2, and $39(8.6 \%)$ in N3; 29 $(6.4 \%)$ in stage I, $107(23.5 \%)$ in stage II, $174(38.2 \%)$ in stage III, and $145(31.9 \%)$ in stage IVa.

\section{Radiotherapy}

Patients were immobilized in a supine position using a head to shoulder thermoplastic mask vacuum pad. Simulation CT images were obtained from the head to $2 \mathrm{~cm}$ below the sternoclavicular joint (slice thickness, $3 \mathrm{~mm}$ ). Target volumes were delineated using the RTOG 0225 and RTOG 0615 RT protocol ${ }^{12}$, 13. The gross tumor volume (GTV) included the primary tumor (GTV-P) and metastatic lymph nodes (GTV-N). The CTV70 included the GTV-P and lymph nodes with extracapsular spread plus a $5 \mathrm{~mm}$ margin. The CTV59.4 included the entire nasopharynx, retropharyngeal lymph nodal regions, clivus, skull base, pterygoid fossae, parapharyngeal space, inferior sphenoid sinus, posterior third of nasal cavity and maxillary sinuses, high risk nodal regions. The CTV50.4 included the low risk nodal regions.

The planning target volumes (PTVs) were defined as the CTVs with $3 \mathrm{~mm}$ margins. The PTV70 received $70 \mathrm{~Gy}$ in 33 fractions at 2.12 Gy per fraction. The small volume lymph nodes received $63 \mathrm{~Gy}$ in 33 fractions at 1.9 Gy per fraction. The PTV59.4 received $59.4 \mathrm{~Gy}$ in 33 fractions at $1.8 \mathrm{~Gy}$ per fraction. The PTV50.4 received 50.4 Gy in 28 fractions at 1.8 Gy per fraction. RT was delivered over one fraction daily, 5 days per week. Dose optimization and evaluation were performed according to the RTOG 0225 and RTOG 0615 RT protocols. Critical normal structures, including the brainstem, spinal cord, optic nerves, optic chiasm, temporal lobes, hypophysis, lens, eyeballs, inner ears, parotid glands, temporomandibular (T-M) joints, mandible, oral cavity and glottic larynx were outlined. Dose constraints for normal structures were defined and evaluated according to the RTOG 0225 and RTOG 0615 RT protocols.

Boost irradiation was provided if necessary to the primary tumor or metastatic lymph nodes with obvious residual disease after RT, and did not exceed 16 Gy. In total, 50 patients $(50 / 455,11 \%)$ received boost irradiation, including 34 patients treated by brachytherapy, two patients treated by IMRT and 14 patients treated by two-dimensional RT.

\section{Chemotherapy}

In total, $91.1 \%(288 / 316)$ patients with stage III-IVb NPC received chemotherapy: 176 received 
induction chemotherapy + concurrent chemotherapy, 78 received concurrent chemotherapy, 33 received induction chemotherapy and one patient received adjuvant chemotherapy. Induction chemotherapy or adjuvant chemotherapy consisted of cisplatin (80 $\left.\mathrm{mg} / \mathrm{m}^{2}\right)$ and fluorouracil $\left(1000 \mathrm{mg} / \mathrm{m}^{2}\right.$ daily for 4 days); docetaxel $\left(75 \mathrm{mg} / \mathrm{m}^{2}\right)$ and cisplatin (75 $\left.\mathrm{mg} / \mathrm{m}^{2}\right)$; or a triplet of docetaxel $\left(60 \mathrm{mg} / \mathrm{m}^{2}\right)$, cisplatin $\left(60 \mathrm{mg} / \mathrm{m}^{2}\right)$ and fluorouracil $\left(800 \mathrm{mg} / \mathrm{m}^{2}\right.$ daily for 4 days) every 3 weeks for 2-3 cycles. Concurrent chemotherapy was cisplatin given every 3 weeks $(100$ $\left.\mathrm{mg} / \mathrm{m}^{2}\right)$ or weekly $\left(40 \mathrm{mg} / \mathrm{m}^{2}\right)$ during RT. Patients with advanced-stage disease did not receive chemotherapy due to patient refusal, advanced age, hepatitis, severe diabetes, heart disease, inadequate renal function or financial issues. In the event of documented relapse or persistent disease, salvage treatments including RT, surgery or chemotherapy were provided when appropriate.

\section{Follow up and statistical analysis}

Patients were assessed every three months during first two years after RT, and every six months thereafter. Imaging methods including a MRI scan of the nasopharynx and neck, chest $X$-ray and abdominal sonography were recommended at 3-4 months after RT, every six months during the first three years, every year during the first 3-5 years, and as necessary thereafter. Chemotherapy-related toxicities were graded using the Common Terminology Criteria for Adverse Events (CTCAE), version 3.0. Acute and late RT-related toxicities were graded based on the Radiation Morbidity Scoring Criteria of the RTOG. Radiation-induced temporal lobe injury (TLI) was diagnosed as white matter lesions, contrast-enhanced lesions or cysts in the temporal lobe on MRI, using criteria previously described by Zhou et al.20. A diagnosis of radiation-induced cranial nerve palsy (CNP) was based on the patient's history and physical examination, following the criteria described by Lin $e t$ al. ${ }^{21 .}$

Overall survival (OS) was measured from assignment to date of death from any cause. Failure free survival (FFS) was defined as first failure at any site. Local relapse free survival (LRFS), regional relapse free survival (RRFS) and distant metastasis free survival (DMFS) were recorded as first local, regional or remote failure, respectively. Distant metastases were diagnosed according to clinical symptoms, physical examinations and imaging methods consisting of X-ray, abdominal sonography, bone scan, CT, MRI and PET-CT. Locoregional recurrence was diagnosed based on fiberoptic endoscopy, biopsy, MRI and PET-CT.
All analyses were performed using SPSS software version 22.0 (IBM Corporation, Armonk, NY, USA). Actuarial rates were calculated using the Kaplan-Meier method and compared using the log-rank test. Multivariate analyses with the Cox proportional hazards model were used to identify significant independent prognostic factors by backward elimination. The parameters included in the Cox proportional hazards model were: age ( $\leq 45$ vs. $>$ 45 years), sex (male vs. female), chemotherapy (yes vs. no), additional RT boosts (yes vs. no), T category (T1-2 vs. T3-4) and N category (N0-1 vs. N2-3). The criterion for statistical significance was set at $\alpha=0.05 ; P$-values were based on two-sided tests.

\section{Results}

\section{Survival}

Four-hundred and thirty-nine (439/455, 96.5\%) patients received regular follow-up until death or latest scheduled assessment. Last follow-up was March 3, 2017; median follow-up was 53 months (range, 2-83 months). In all, 90 patients died and 90 experienced treatment failure (Figure 1). Estimated four-year OS, FFS, LRFS, RRFS and DMFS were $83.8 \%, 80.5 \%, 94.3 \%, 96.7 \%$ and $85.8 \%$ (Figure $2-3$ ). $\mathrm{T}$ category and $\mathrm{N}$ category were significant prognostic factors for OS, FFS, RRFS and DMFS; T category was a significant prognostic factor for LRFS (Table 1).

\section{- Local relapse $\quad$ Regional relapse \\ - Local relapse+regional relapse Local relapse+metastasis \\ - Regional relapse+metastasis Metastasis}

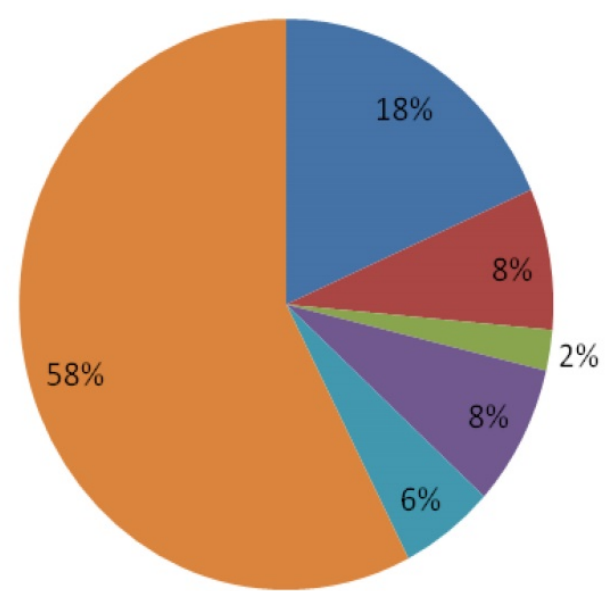

Figure 1. Failure patterns for the 90 patients with nasopharyngeal carcinoma who experienced treatment failure. 


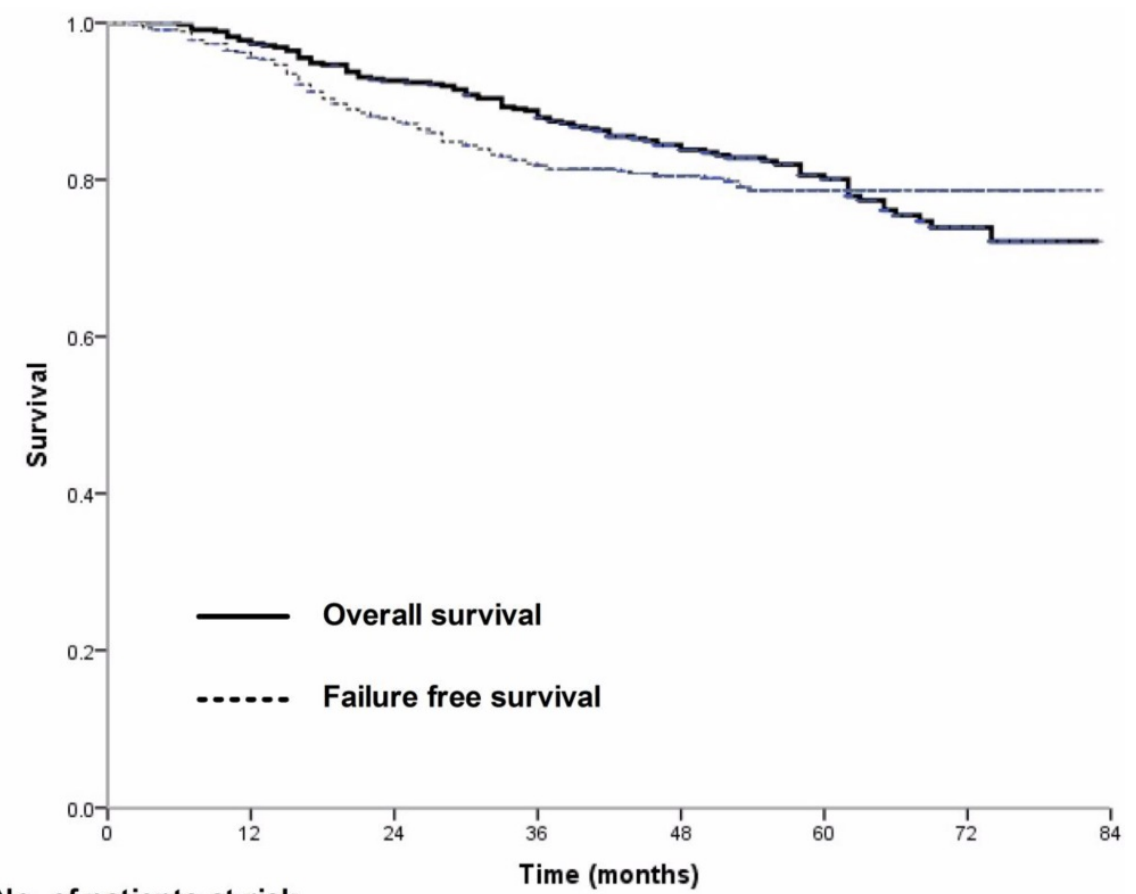

No. of patients at risk

Time (months)

$\begin{array}{lllllllll}\text { OS } & 455 & 437 & 412 & 384 & 259 & 155 & 53 & 0 \\ \text { FFS } & 455 & 423 & 380 & 346 & 240 & 139 & 50 & 0\end{array}$

Figure 2. Overall survival and failure-free survival for 455 patients with nasopharyngeal carcinoma treated using IMRT based on the RTOG 0225 and RTOG 0615 protocols.

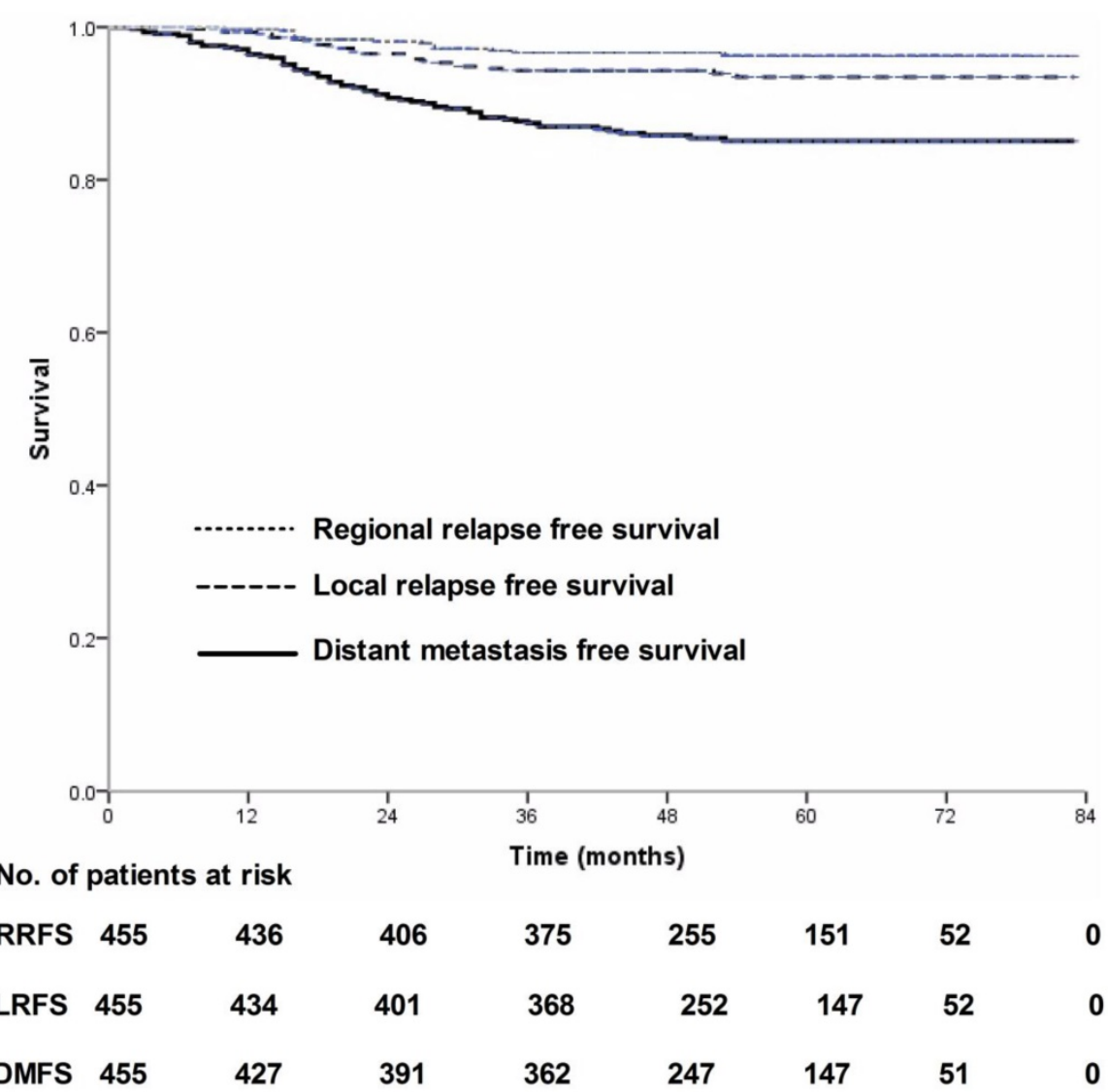

Figure 3. Local relapse-free survival, regional relapse-free survival and distant metastasis-free survival for 455 patients with nasopharyngeal carcinoma treated using IMRT based on the RTOG 0225 and RTOG 0615 protocols. 
Table 1. Multivariate analyses of prognostic factors in 455 patients with nasopharyngeal carcinoma

\begin{tabular}{lllll}
\hline Endpoint & Variable & HR & $95 \%$ CI & $P$-value \\
\hline OS & T category & 2.317 & $1.431-3.753$ & 0.001 \\
& N category & 2.393 & $1.581-3.623$ & $<0.001$ \\
\multirow{2}{*}{ FFS } & Sex & 1.767 & $0.980-3.186$ & 0.058 \\
& T category & 2.083 & $1.304-3.326$ & 0.002 \\
LRFS & N category & 2.272 & $1.501-3.439$ & $<0.001$ \\
RRFS & T category & 9.334 & $2.205-39.510$ & 0.002 \\
& T category & 0.341 & $0.116-0.998$ & 0.050 \\
DMFS & N category & 7.323 & $2.329-23.029$ & 0.001 \\
& T category & 2.181 & $1.236-3.848$ & 0.007 \\
& N category & 2.727 & $1.662-4.474$ & $<0.001$
\end{tabular}

Abbreviations: HR, hazard ratio; CI, confidence interval; OS, overall survival; FFS, failure free survival; LRFS, local relapse free survival; RRFS, regional relapse free survival; DMFS, distant metastasis-free survival.

\section{Loco-regional relapse}

Of the 26 cases of local relapse, 18 (18/26, 69.2\%) were in-field, five $(5 / 26,19.2 \%)$ were marginal-field and three $(3 / 26,11.5 \%)$ were out-of-field; of these, one patient had T1 NPC, one T2, $16 \mathrm{~T} 3$, and eight T4. T category (T1-2 vs. T3-4) was significantly associated with LRFS $(P<0.001)$.

Of the 15 cases of regional relapse, ten (10/15, $66.7 \%)$ were in-field, one $(1 / 15,6.7 \%)$ was marginal-field and four $(4 / 15,26.7 \%)$ were out-of-field. Of the 15 patients who experienced regional relapse, four had N1 NPC, five N2 and six N3. N category (N0-1 vs. N2-3) was significantly associated with RRFS $(P<0.001)$.

\section{Acute and late toxicities}

During the radiotherapy phase, 16 patients $(16 / 455,3.5 \%)$ experienced grade 4 toxicities and 190 (190/455, 41.8\%) experienced grade 3 toxicities. The most common acute toxicity was mucositis (with 124/455 [27.2\%] experiencing grade 3-4 mucositis). The second most common acute grade 3-4 toxicity was leukopenia/neutropenia (59/455, 13\%). Acute toxicities are listed in Table 2.

Forty-five patients (46/455, 10.1\%) experienced serious late toxicities, including MRI-detected radiation-induced TLN, radiation-induced CNP and mandible necrosis, and grade $\geq 3$ hearing loss, xerostomia and soft tissue damage. The most common late toxicity was MRI-detected radiation-induced TLN $(31 / 455,6.8 \%)$, followed by radiation-induced CNP $(7 / 455,1.5 \%)$. Late toxicities are listed in Table 2.

\section{Discussion}

The RTOG 0225 and RTOG 0615 protocols for NPC provided good treatment outcomes and acceptable RT-related toxicities in patients from the non-endemic regions ${ }^{12}$. However, it is imperative to apply these protocols to patients from the areas of China where NPC is endemic, and further assess long-term survival and late toxicities. To the best of our knowledge, this is the first study to evaluate the RTOG 0225 and RTOG 0615 RT protocol in patients from a region of China where is NPC endemic. Overall, local and regional controls were excellent and RT-related toxicities were acceptable. Metastasis remained the main treatment failure and in-field failures were the major loco-regional failure pattern.

Table 2. Acute and late toxicities for 455 patients with nasopharyngeal carcinoma treated by intensity-modulated radiation therapy

\begin{tabular}{llll}
\hline Toxicities & Grade 3 & Grade 4 & Grade 5 \\
\hline Acute toxicities & & & \\
Mucositis (radiation-related) & $118(25.9 \%)$ & $6(1.3 \%)$ & 0 \\
Skin reaction (radiation-related) & $2(0.4 \%)$ & 0 & 0 \\
Leukopenia/neutropenia & $55(12.1 \%)$ & $4(0.9 \%)$ & 0 \\
Thrombocytopenia & $19(4.2 \%)$ & $9(2.0 \%)$ & 0 \\
Anemia & $3(0.7 \%)$ & 0 & 0 \\
Nausea/vomiting & $22(4.8 \%)$ & 0 & 0 \\
Weight loss & $9(2.0 \%)$ & 0 & 0 \\
Any acute toxicities & $190(41.8 \%)$ & $16(3.5 \%)$ & 0 \\
Late toxicities & & & \\
TLN & $31(6.8 \%)$ & 0 & 0 \\
CNP & $7(1.5 \%)$ & 0 & 0 \\
Hearing loss & $3(0.7 \%)$ & 0 & 0 \\
Xerostomia & $3(0.7 \%)$ & 0 & 0 \\
Soft tissue damage & $1(0.2 \%)$ & 0 & 0 \\
Mandible necrosis & $3(0.7 \%)$ & 0 & 0 \\
Any late toxicities & $46(10.1 \%)$ & 0 & 0 \\
\hline
\end{tabular}

Abbreviations: TLN: temporal lobe injury; CNP: cranial nerve palsy.

\section{Survival after IMRT based on the RTOG protocols}

In this study, estimated 4-year OS, LRFS, RRFS and DMFS were $83.8 \%, 94.3 \%, 96.7 \%$ and $85.8 \%$, respectively. In the RTOG 0225 trial, 2-year OS, local progression-free survival (PFS), regional PFS and DMFS were $80.2 \%, 92.6 \%, 90.8 \%$ and $84.7 \%$, respectively 12 . Our long-term survival outcomes seem slightly superior, which may be related to the fact $8 \%$ of patients in the RTOG 0225 trial had keratinizing NPC while all patients in this study had non-keratinizing NPC; these histological types have different prognoses ${ }^{22}$.

We further compared the survival outcomes in this study with patients treated using the RTV-IMRT protocols in China. Lin et al. reported estimated 3-year OS, LRFS, RRFS and DMFS were $90 \%, 95 \%, 98 \%$ and $90 \%$, respectively 18 . Sun et al. reported estimated 5-year disease specific survival, LRFS, RRFS and DMFS were $84.7 \%, 91.8 \%, 96.4 \%$ and $84.6 \%$, respectively ${ }^{23}$. Thus, the local control and regional control rates for patients treated using either the RTOG IMRT protocols or RTV-IMRT protocols are all very high. Metastasis was the main pattern of treatment failure in all studies, reflecting the change 
in failure patterns in NPC after the widespread introduction of IMRT 24, 25 .

\section{Loco-regional relapse after IMRT based on the RTOG protocols}

In this study, among patients with loco-regional relapse, 18 patients $(69.2 \%)$ suffered in-field local relapse failures and ten $(66.7 \%)$ suffered in-field regional relapse failures. In the RTOG 0225 trial, seven patients experienced loco-regional relapse, in whom some regional failures resulted from incorrect contouring of level V; the causes of loco-regional relapse were not reported ${ }^{12}$. In the study by $\mathrm{Ng}$ et al., of the 16 local failures, 13 were considered in-field failures; of the nine regional failures, eight were considered in-field failures ${ }^{14}$. Loco-regional relapse was significantly associated with advanced clinical stage in this study.

A similar pattern of loco-regional relapse failure in patients treated using the RTV-IMRT protocols was also observed in other studies. In the study by Kong et al., of the 22 loco-regional failures, 16 (64\%) occurred in-field, three $(12 \%)$ were marginal and three $(12 \%)$ were out-of-field ${ }^{26}$. Xiao et al. reported that all local recurrences in patients treated using the RTV-IMRT protocols were in-field failures ${ }^{27}$. Therefore, in-field failures represent the major pattern of loco-regional failure in patients with NPC treated using either the RTOG IMRT protocols or RTV-IMRT protocols. With the development of precise radiotherapy techniques, radioresistance may now be the major cause of local-regional failure. Tumor hypoxia is common in NPC and contributes to radioresistance ${ }^{28-30}$.

\section{Acute and late toxicities of IMRT based on the RTOG protocol}

In this study, during the RT phase, the most common acute toxicity was mucositis (grade 3-4, $27.2 \%)$. In the RTOG 0225 trial, the rates of acute 3 and 4 mucositis/stomatitis toxicities were $36.8 \%$ and $4.4 \%$ 12. The incidence of acute 3-4 mucositis was higher in the RTOG 0225 trial than this trial. One possible reason may be that $87.7 \%$ of patients received all three cycles of concurrent chemotherapy in the RTOG 0225 trial, which may aggravate acute mucositis 31,32 . In RTV-IMRT studies, the incidence of acute grade 3-4 stomatitis ranged from $18.5 \%-40.1 \% 16,33-35$. Compared to the RTOG IMRT, the RTV-IMRT has a smaller target volume, which may reduce the incidence of acute stomatitis. However, the single dose to the GTV is higher in the RTV-IMRT than RTOG IMRT (2.2-2.3 vs. $2.12 \mathrm{~Gy}$ ), which may affect acute stomatitis.

Radiation-induced TLN was the most common neurological complication in this study (6.8\%); similarly, Zeng et al. reported $7.5 \%$ (59/789) of patients developed temporal lobe injury ${ }^{36}$. However, no patient developed radiation-induced TLN in the RTOG 0225 trial. One possible reason may be that the rate of MRI-detected TLN was higher than the incidence based on the patient's history and physical examination. Most patients with radiation-induced TLN were either asymptomatic or only had mild symptoms and no localizing signs ${ }^{37}$. IMRT can significantly reduce the incidence of radiation-induced TLN compared to 2-DRT, as IMRT minimizes unnecessary doses to the temporal lobes ${ }^{20}$. Apart from RT technology, fractional dose also has a significant impact on the risk of radiation-induced TLN 37.

This study demonstrated the RTOG 0225 and RTOG 0615 IMRT protocols are feasible for patients from the areas of China where NPC is endemic. However, this was a retrospective study of consecutive patients; therefore, different induction/adjuvant chemotherapy and concurrent chemotherapy regimens were employed, which may affect survival outcomes and treatment-related toxicities in addition to IMRT protocol ${ }^{38,39}$. Moreover, this was a retrospective study of single-institution data, which needs to be confirmed by multicenter studies.

\section{Conclusions}

This study demonstrates the RTOG 0225 and RTOG 0615 IMRT protocols provide encouraging survival outcomes and acceptable RT-related toxicities in patients with NPC from a region of China where is NPC endemic. Distant metastasis is still the biggest obstacle to treatment success; further studies are required to address this issue in NPC.

\section{Abbreviations}

IMRT: intensity-modulated radiation therapy; NPC: nasopharyngeal carcinoma; OS: overall survival; FFS: failure free survival; LRFS: local relapse free survival; RRFS: regional relapse free survival; DMFS: distant metastasis free survival; RT: radiotherapy; 2-DRT: two-dimensional RT; 3-DCRT: three-dimensional conformal RT; RTOG: Radiation Therapy Oncology Group; RTV-IMRT: reduced-target-volume IMRT; CTV: clinical target volume; MRI: magnetic resonance imaging; AJCC: the American Joint Commission on Cancer staging system; GTV: gross tumor volume; GTV-P: primary tumor; GTV-N: metastatic lymph nodes; PTVs: planning target volumes; T-M: temporo-mandibular; CTCAE: Common Terminology Criteria for Adverse Events; TLI: temporal lobe injury; CNP: cranial nerve palsy; PFS: progression-free survival. 


\section{Acknowledgements}

This work was supported by grants from the National Natural Science Foundation of China (No. 1473233), the Science Foundation from the Sci-Tech Office of Guangdong Province, China (No. 2016A020215083) and the Science Foundation from the Sci-Tech Office of Foshan City, China (No. 2015АB00294).

\section{Competing Interests}

The authors have declared that no competing interest exists.

\section{References}

1. Cancer incidence in five continents. Volume VIII. IARC Sci Publ. 2002: 1-781.

2. Chen Y, Sun Y, Liang SB, et al. Progress report of a randomized trial comparing long-term survival and late toxicity of concurrent chemoradiotherapy with adjuvant chemotherapy versus radiotherapy alone in patients with stage III to IVB nasopharyngeal carcinoma from endemic regions of China. Cancer. 2013;119: 2230-8.

3. Chen $\mathrm{Y}, \mathrm{Hu} \mathrm{XF}$, Wang $\mathrm{Y}$, et al. Is maximum primary tumor diameter still a prognostic factor in patients with nasopharyngeal carcinoma treated using intensity-modulated radiotherapy? BMC Cancer. 2015;15: 305.

4. Al-Sarraf M, LeBlanc M, Giri PG, et al. Chemoradiotherapy versus radiotherapy in patients with advanced nasopharyngeal cancer: phase III randomized Intergroup study 0099. J Clin Oncol. 1998;16: 1310-7.

5. Langendijk JA, Leemans CR, Buter J, Berkhof J, Slotman BJ. The additional value of chemotherapy to radiotherapy in locally advanced nasopharyngeal carcinoma: a meta-analysis of the published literature. J Clin Oncol. 2004;22: 4604-12.

6. Chen L, Hu CS, Chen XZ, et al. Concurrent chemoradiotherapy plus adjuvant chemotherapy versus concurrent chemoradiotherapy alone in patients with locoregionally advanced nasopharyngeal carcinoma: a phase 3 multicentre randomised controlled trial. Lancet Oncol. 2012;13: 163-71.

7. Blanchard P, Lee A, Marguet S, et al. Chemotherapy and radiotherapy in nasopharyngeal carcinoma: an update of the MAC-NPC meta-analysis. Lancet Oncol. 2015;16: 645-55.

8. Lee $\mathrm{N}$, Xia P, Quivey JM, et al. Intensity-modulated radiotherapy in the treatment of nasopharyngeal carcinoma: an update of the UCSF experience. Int J Radiat Oncol Biol Phys. 2002;53: 12-22.

9. Lai SZ, Li WF, Chen L, et al. How does intensity-modulated radiotherapy versus conventional two-dimensional radiotherapy influence the treatment results in nasopharyngeal carcinoma patients? Int J Radiat Oncol Biol Phys. 2011;80: 661-8.

10. Peng G, Wang $\mathrm{T}$, Yang $\mathrm{KY}$, et al. A prospective, randomized study comparing outcomes and toxicities of intensity-modulated radiotherapy vs. conventional two-dimensional radiotherapy for the treatment of nasopharyngeal carcinoma. Radiother Oncol. 2012;104: 286-93.

11. Huang TL, Chien CY, Tsai WL, et al. Long-term late toxicities and quality of life for survivors of nasopharyngeal carcinoma treated with intensity-modulated radiotherapy versus non-intensity-modulated radiotherapy. Head Neck. 2016;38 Suppl 1: E1026-32.

12. Lee N, Harris J, Garden AS, et al. Intensity-modulated radiation therapy with or without chemotherapy for nasopharyngeal carcinoma: radiation therapy oncology group phase II trial 0225. J Clin Oncol. 2009;27: 3684-90.

13. Lee NY, Zhang Q, Pfister DG, et al. Addition of bevacizumab to standard chemoradiation for locoregionally advanced nasopharyngeal carcinoma (RTOG 0615): a phase 2 multi-institutional trial. Lancet Oncol. 2012:13: 172-80.

14. Ng WT, Lee MC, Hung WM, et al. Clinical outcomes and patterns of failure after intensity-modulated radiotherapy for nasopharyngeal carcinoma. Int J Radiat Oncol Biol Phys. 2011;79: 420-8.

15. Setton J, Han J, Kannarunimit D, et al. Long-term patterns of relapse and survival following definitive intensity-modulated radiotherapy for non-endemic nasopharyngeal carcinoma. Oral Oncol. 2016;53: 67-73.

16. Lin S, Pan J, Han L, Zhang X, Liao X, Lu JJ. Nasopharyngeal carcinoma treated with reduced-volume intensity-modulated radiation therapy: report on the 3-year outcome of a prospective series. Int J Radiat Oncol Biol Phys. 2009;75: $1071-8$.

17. Zheng $\mathrm{Y}, \mathrm{Han} \mathrm{F}$, Xiao $\mathrm{W}$, et al. Analysis of late toxicity in nasopharyngeal carcinoma patients treated with intensity modulated radiation therapy. Radiat Oncol. 2015;10: 17

18. Lin YW, Chen CC, Lin LC, Lee SP. The impact of reduced-volume, intensity-modulated radiation therapy on disease control in nasopharyngeal carcinoma. PLoS One. 2015;10: e0125283.
19. Edge SB, Compton CC. The American Joint Committee on Cancer: the 7th edition of the AJCC cancer staging manual and the future of TNM. Ann Surg Oncol. 2010;17: 1471-4.

20. Zhou GQ, Yu XL, Chen M, et al. Radiation-induced temporal lobe injury for nasopharyngeal carcinoma: a comparison of intensity-modulated radiotherapy and conventional two-dimensional radiotherapy. PLoS One. 2013;8: e67488.

21. Kong L, Lu JJ, Liss AL, et al. Radiation-induced cranial nerve palsy: a cross-sectional study of nasopharyngeal cancer patients after definitive radiotherapy. Int J Radiat Oncol Biol Phys. 2011;79: 1421-7.

22. Marks JE, Phillips JL, Menck HR. The National Cancer Data Base report on the relationship of race and national origin to the histology of nasopharyngeal carcinoma. Cancer. 1998;83: 582-8.

23. Sun $X$, Su S, Chen $C$, et al. Long-term outcomes of intensity-modulated radiotherapy for 868 patients with nasopharyngeal carcinoma: an analysis of survival and treatment toxicities. Radiother Oncol. 2014;110: 398-403.

24. Qiu WZ, Huang PY, Shi JL, Xia HQ, Zhao C, Cao KJ. Neoadjuvant chemotherapy plus intensity-modulated radiotherapy versus concurrent chemoradiotherapy plus adjuvant chemotherapy for the treatment of locoregionally advanced nasopharyngeal carcinoma: a retrospective controlled study. Chin J Cancer. 2016;35: 2

25. Liu X, Tang LL, Du XJ, et al. Changes in Disease Failure Risk of Nasopharyngeal Carcinoma over Time: Analysis of 749 Patients with Long-Term Follow-Up. J Cancer. 2017;8: 455-9.

26. Kong F, Ying H, Du C, et al. Patterns of local-regional failure after primary intensity modulated radiotherapy for nasopharyngeal carcinoma. Radiat Oncol. 2014;9: 60.

27. Xiao WW, Huang SM, Han F, et al. Local control, survival, and late toxicities of locally advanced nasopharyngeal carcinoma treated by simultaneous modulated accelerated radiotherapy combined with cisplatin concurrent chemotherapy: long-term results of a phase 2 study. Cancer. 2011;117: 1874-83.

28. Hong B, Lui VW, Hashiguchi M, Hui EP, Chan AT. Targeting tumor hypoxia in nasopharyngeal carcinoma. Head Neck. 2013;35: 133-45.

29. Yeh SH, Liu RS, Wu LC, et al. Fluorine-18 fluoromisonidazole tumour to muscle retention ratio for the detection of hypoxia in nasopharyngeal carcinoma. Eur J Nucl Med. 1996;23: 1378-83.

30. Nordsmark M, Overgaard M, Overgaard J. Pretreatment oxygenation predicts radiation response in advanced squamous cell carcinoma of the head and neck. Radiother Oncol. 1996;41: 31-9.

31. Chen QY, Wen YF, Guo L, et al. Concurrent chemoradiotherapy vs radiotherapy alone in stage II nasopharyngeal carcinoma: phase III randomized trial. J Natl Cancer Inst. 2011;103: 1761-70.

32. Lee AW, Tung SY, Chua DT, et al. Randomized trial of radiotherapy plus concurrent-adjuvant chemotherapy vs radiotherapy alone for regionally advanced nasopharyngeal carcinoma. J Natl Cancer Inst. 2010;102: 1188-98

33. Kong FF, Ying H, Du CR, Huang S, Zhou JJ, Hu CS. Effectiveness and toxicities of intensity-modulated radiation therapy for patients with T4 nasopharyngeal carcinoma. PLoS One. 2014;9: e91362.

34. Tao CJ, Lin L, Zhou GQ, et al. Comparison of long-term survival and toxicity of cisplatin delivered weekly versus every three weeks concurrently with intensity-modulated radiotherapy in nasopharyngeal carcinoma. PLoS One. 2014;9: e110765.

35. Sun Y, Li WF, Chen NY, et al. Induction chemotherapy plus concurrent chemoradiotherapy versus concurrent chemoradiotherapy alone in locoregionally advanced nasopharyngeal carcinoma: a phase 3, multicentre, randomised controlled trial. Lancet Oncol. 2016;17: 1509-20.

36. Zeng L, Tian YM, Sun XM, et al. Late toxicities after intensity-modulated radiotherapy for nasopharyngeal carcinoma: patient and treatment-related risk factors. Br J Cancer. 2014:110: 49-54.

37. Lee AW, Kwong DL, Leung SF, et al. Factors affecting risk of symptomatic temporal lobe necrosis: significance of fractional dose and treatment time. Int J Radiat Oncol Biol Phys. 2002;53: 75-85.

38. Guan Y, Liu S, Wang HY, et al. Long-term outcomes of a phase II randomized controlled trial comparing intensity-modulated radiotherapy with or without weekly cisplatin for the treatment of locally recurrent nasopharyngeal carcinoma. Chin J Cancer. 2016;35: 20.

39. Peng H, Chen L, Zhang J, et al. Induction Chemotherapy Improved Long-term Outcomes of Patients with Locoregionally Advanced Nasopharyngeal Carcinoma: A Propensity Matched Analysis of 5-year Survival Outcomes in the Era of Intensity-modulated Radiotherapy. J Cancer. 2017;8: 371-7. 